Seventh Meeting, 9th June 1899.

Alexander Morgan, Esq., M.A., D.Sc., President, in the Chair.

\title{
Systems of Circles analogous to Tucker Circles."
}

By J. A. Third, M.A.

I.

\section{Systems of Six-Point Circles connected with the Triangle. General Theorens. $\dagger$}

1. If a circle meets the sides $\mathrm{BC}, \mathrm{CA}, \mathrm{AB}$ of a triangle (Fig. 6) in the pairs of points $\mathrm{I}_{2}$ and $l, \mathrm{M}$ and $m, \mathrm{~N}$ and $n$ respectively, it is obvious that the pairs of connectors $\mathrm{M} n$ and $m \mathrm{~N}, \mathrm{~N} l$ and $n \mathrm{~L}$, $\mathrm{L} m$ and $l \mathrm{M}$ are antiparallel with respect to the angles $\mathrm{A}, \mathrm{B}, \mathrm{C}$ respectively.

Conversely, by a modification of a theorem of Poncelet's, if the above-mentioned pairs are antiparallel with respect to $\mathrm{A}, \mathrm{B}, \mathrm{C}$ respectively, the six points $\mathrm{L}, l, \mathrm{M}, m, \mathrm{~N}, n$ lie on a circle; for if not, $\mathrm{M}, n, \mathrm{~N}, m$ and $\mathrm{N}, l, \mathrm{~L}, n$ and $\mathrm{L}, m, \mathrm{M}, l$ would lie on three distinct circles, and consequently $\mathrm{BC}, \mathrm{CA}, \mathrm{AB}$, their radical axes, would be concurrent.

* Mr Third's Papers were received on 29th March. Sections I. and II. of the First Puper, in a different form, were read at the November and December Meetings.

+ Since writing this paper I have found that a considerable number of the theorems contained in Part I. are given in two English memoirs, which I had somehow overlooked, viz., "The Relations of the Intersections of a Circle with a Triangle," by H. M. Taylor, Proc. of the London Math. Soc., Vol. XV., pp. 122-139, in which the treatment is mainly analytical, and "Some Geometrical Proofs of Theorems connected with the Inseription of a Triangle of constant Form in a given Triangle," by M. Jenkins, Quarterly Journal, Vol. XXI., pp. 84-89. Among the theorems in Part I. which seem to be new, the most important, perhaps, are those referring to the connection between the S.point and the angles of a system, and to the relation of the systems to coaxal systems. The special cases of Arts. 19 and 22, Part II., are also referred to in the above-mentioned papers, but in the former of these cases the $\mathbf{S}$-point is not determined. 
2. It follows at once from Pascul's theorem that in the figure of the preceding article (Fig. 6) the following are triads of collinear points

$A$, and the points of intersection of the pairs $\mathrm{L} m, \mathrm{~N} l$ and $l \mathrm{M}, n \mathrm{~L}$;

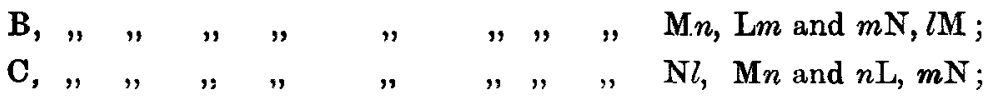

and also that the three lines on which these triads of points lie are concurrent. Let the point of concurrence be denoted by $\mathbf{S}$.

It is obvious that by joining the six points in which the circle meets the sides of the triangle in all possible ways by chords passing from side to side, we obtain, in general, three other points of concurrence similar to $\mathrm{S}$.

3. If thrce lines be drawn parallel to $\mathrm{M} n, \mathrm{~N} l, \mathrm{Lm}$ (Fig. 6) meeting $\mathrm{CA}, \mathrm{AB}$ in $\mathrm{M}^{\prime}, n^{\prime} ; \mathrm{AB}, \mathrm{BC}$ in $\mathrm{N}^{\prime}, l^{\prime} ; \mathrm{BC}, \mathrm{CA}$ in $\mathrm{L}^{\prime}, m^{\prime}$ respectively, and intersecting two and two on $\mathrm{SC}, \mathrm{SA}, \mathrm{SB}$, the six points $\mathrm{L}^{\prime}, l^{\prime}, \mathrm{M}^{\prime}, m^{\prime}, \mathrm{N}^{\prime}, n^{\prime}$ also lie on a circle.

Demonstration. Let $\mathbf{L} m, \mathbf{N} l$ intersect in $\mathrm{P}$, and $\mathbf{L}^{\prime} m^{\prime}, \mathbf{N}^{\prime} l^{\prime}$ in $\mathbf{P}^{\prime}$. Then $\mathbf{A N} / \mathbf{A N}=\mathbf{A P} / \mathbf{A P} \mathbf{P}^{\prime}=\mathbf{A} m / \mathbf{A} m^{\prime}$. Therefore $m \mathbf{N}, m^{\prime} \mathbf{N}^{\prime}$ are parallel. Therefore $\mathrm{M}^{\prime} n^{\prime}, m^{\prime} \mathrm{N}^{\prime}$ are antiparallel with respect to the angle $A$. Similarly it may be shown that $\mathrm{N}^{\prime} l^{\prime}, n^{\prime} \mathrm{L}^{\prime}$ are antiparallel with respect to the angle $\mathrm{B}$, and that $\mathrm{J}^{\prime} \mathrm{m}^{\prime}, l^{\prime} \mathrm{M}^{\prime}$ are antiparallel with respect to the angle $\mathrm{C}$. Therefore, by (1), L', $l^{\prime}, \mathrm{M}^{\prime}, m^{\prime}, \mathrm{N}^{\prime}, n^{\prime}$ are concyclic.

By drawing a system of parallels to $\mathrm{M} n, \mathrm{~N} l, \mathrm{Lm}$ as above, we obtain a system of six-point circles of which the original circle is one. Such a systen may be called, in the meantime, an S-point system. The chords $\mathrm{M} n, \mathrm{~N} l, \mathrm{Lm}$ of the circle which is regarded as the original circle of the system may be called the directive chords of the system.

It is obvious that the same s-point system would be obtained by drawing parallels (intersecting two and two on $\mathrm{SC}, \mathrm{SA}, \mathrm{SB}$ ) to $m \mathrm{~N}, n \mathrm{~L}, l \mathrm{M}$, i.e., by drawing antiparallels to $\mathrm{M} n, \mathrm{~N} l, \mathrm{~L} m$ with respect to the angles $A, B, C$ respectively. In other words $m \mathrm{~N}, n \mathrm{~L}, l \mathrm{M}$ may equally well with $\mathrm{M} n, \mathrm{~N} l, \mathrm{~L} m$ be regarded as directive chords of the system. 
The following particular cases may be noticed.

(i) Two circles of the system are, in general, obtained by drawing (1) parallels and (2) antiparallels to the directive chords, so as to cointersect in $\mathbf{S}$.

(ii) Two circles of the system are, in generul, determined by drawing through any point $\mathbf{P}^{\prime}$ on SA (or SB or SC) (1) parallels and (2) antiparallels to the two directive chords which meet on that line; but in the case where $\mathbf{P}^{\prime}$ also lies on $\mathrm{BC}$ (or $\mathrm{CA}$ or $\mathrm{AB}$ ), these two circles coalesce and touch $\mathrm{BC}$ (or $\mathrm{CA}$ or $\mathrm{AB}$ ) at $\mathrm{P}^{\prime}$.

(iii) If from $A$, lines $\mathrm{AX}, \mathbf{A X}$ be drawn parallel to the directive chords which intersect on SA, to meet the opposite side BC, and if from $B$ and $C$ analogous pairs of lines $B Y, B^{\prime}$ and $C Z, C Z^{\prime}$ be drawn to meet the opposite sides, the circumcircles of the triangles $A X X^{\prime}, B Y Y^{\prime}$ and $\mathrm{CZZ}^{\prime}$ belong to the system.

It is obvious that if we take in succession, as S-point, each of the three other points of concurrence referred to in Art. 2 as being obtainable in connection with the original circle, we obtain three other S-point systems. Thus, in general, every six-point circle connected with a triangle, belongs to four different $S$-point systems. It will be noticed that while each circle of a system has, in general, four S-points, the circles of the system have one and only one S-point in common.

4. If $x, y, z$ be the trilinear courdinates of $s$, and $\alpha, \beta, \gamma$ respectively denote the angles $A n \mathrm{M}, \mathrm{B} l \mathrm{~N}, \mathrm{C} m \mathrm{~L}$ which the directive chords make with the sides of $\mathrm{ABC}$, (Fig. 6) we have the following relations :

$$
\text { (i) } \begin{aligned}
& x / \operatorname{cosec} \alpha \operatorname{cosec}(\mathbf{A}+\alpha) \\
= & y / \operatorname{cosec} \beta \operatorname{cosec}(\mathbf{B}+\beta) \\
= & \boldsymbol{z} / \operatorname{cosec} \gamma \operatorname{cosec}(\mathbf{C}+\gamma) ;
\end{aligned}
$$

and (ii) $u+\beta+\gamma=\pi$.

Demonstration. Let $\mathrm{M}^{\prime \prime} n^{\prime \prime}, \mathrm{N}^{\prime \prime} l^{\prime \prime}, \mathrm{L}^{\prime \prime} \mathrm{m}^{\prime \prime}$ be drawn through $\mathrm{s}$ parallel to $\mathrm{I} n, \mathrm{~N} l, \mathrm{~L} m$ respectively, and meeting the sides of $\mathrm{ABC}$ as in the figure. Then, by Art. 3 (i), $\mathrm{L}^{\prime \prime}, l^{\prime \prime}, \mathrm{M}^{\prime \prime}, m^{\prime \prime}, \mathrm{N}^{\prime \prime}, n^{\prime \prime}$ lie on a circle of the system. Therefore

$$
\mathrm{M}^{\prime \prime} \mathrm{S} . \mathrm{S} u^{\prime \prime}=\mathrm{N}^{\prime \prime} \mathrm{S} . \mathrm{S} l^{\prime \prime}=\mathrm{L} " \mathrm{~S} . \mathrm{S} m^{\prime \prime} \text {. }
$$


But

$\mathrm{M}^{\prime \prime} \mathrm{S}=y \operatorname{cosec} \mathrm{A} \widehat{\mathrm{M}}^{\prime \prime} \mathrm{S}=y \operatorname{cosec}(\mathrm{A}+\alpha)$,

and

$\mathrm{S} n^{\prime \prime}=z \operatorname{cosec} \widehat{\mathrm{An}^{\prime \prime} \mathrm{S}}=z \operatorname{cosec} \alpha$.

Therefore

$\mathbf{M}^{\prime \prime} \mathbf{S} . \mathbf{S} n^{\prime \prime}=y z \operatorname{cosec} \alpha \operatorname{cosec}(\mathbf{A}+a)$.

Similarly it may be shown that

$$
\mathrm{N}^{\prime \prime} \mathrm{S} . \mathbf{S} l^{\prime \prime}=z x \operatorname{cosec} \beta \operatorname{cosec}(\mathrm{B}+\beta) \text {, }
$$

and

$$
\mathbf{L}^{\prime \prime} \mathbf{S} . \mathbf{S} m^{\prime \prime}=x y \operatorname{cosec} \gamma \cos e c(\mathbf{C}+\gamma) \text {. }
$$

Hence the first part of the proposition is established.

Again

$$
\widehat{\mathrm{B} n \mathrm{~L}}=\widehat{\mathrm{B}} \widehat{l} \mathrm{~N}=\beta,
$$

and

$$
\widehat{\mathbf{M}} \widehat{\mathrm{L}}=\widehat{\mathbf{C} m \mathrm{~L}}=\gamma \text {. }
$$

Therefore, the signs being suitably selected,

$$
\alpha+\beta+\gamma=\pi \text {. }
$$

The angles $\alpha, \beta, \gamma($ or $\pi-\mathbf{A}-\alpha, \pi-\mathrm{B}-\beta, \pi-\mathrm{C}-\gamma)$ which the directive chords of an S-point system (or their antiparallels) make with the sides of $A B C$, may be called the angles of the system. The theorem established in the present article enables us to state that the angles of an S-point system and the position of $\mathbf{S}$ implicate euch other, and that if either of these data be given, the system is rleterminate. The theorem is obviously calculated to be of great service in the investigation of particular cases of S-point systems connected with the triangle. The discussion of these, however, is reserved for Part II.

It may be noted that $\mathrm{LL}^{\prime} / \mathrm{L}^{\prime} \mathrm{L}^{\prime \prime}=l l^{\prime} / l^{\prime} l^{\prime \prime}=\mathrm{MM}^{\prime} / \mathrm{M}^{\prime} \mathrm{M}^{\prime \prime}=$ etc., from which it could readily be proved that the centres of the circles of an s-point system are collinear; but this will be established by a shorter method in Art. 8.

5. If $\alpha, \beta, \gamma$ be the angles of an S-point system of circles connected with the triangle $\mathrm{ABC}$, and if on the sides of $\mathrm{ABC}$ (Fig. 7) there be described externally three similar triangles $a \mathrm{BC}, \mathrm{A} b \mathrm{C}, \mathrm{AB} c$, such that

$$
\begin{aligned}
& \widehat{\mathrm{CaB}}=\widehat{\mathrm{CA}} b=\widehat{c A B}=\alpha, \\
& a \widehat{\mathrm{B} C}=\widehat{\mathrm{A}} \widehat{b} \mathbf{C}=\widehat{\mathbf{A B}} c=\beta \text {, } \\
& \text { and } \quad \mathrm{B} \widehat{\mathrm{C}} a=b \widehat{\mathrm{CA}}=\widehat{\mathrm{Bc}} \mathrm{A}=\gamma \text {, }
\end{aligned}
$$

then $\mathrm{A} a, \mathrm{~B} b, \mathrm{C} c$ cointersect in a point,* which $\mathrm{I}$ shall denote by $\Pi$,

* This construction is given by Mr Jenkins in the paper already cited. 
whose trilinear coordinates are

$$
\sin \alpha \operatorname{cosec}(\mathbf{A}+\alpha), \sin \beta \operatorname{cosec}(\mathbf{B}+\beta), \sin \gamma \operatorname{cosec}(\mathbf{C}+\gamma) \text {. }
$$

Also if other three similar triangles $a^{\prime} \mathrm{BC}, \mathrm{A} b^{\prime} \mathrm{C}, \mathrm{ABc}$ be described externally on the sides of $A B C$, such that

and

$$
\begin{aligned}
& \widehat{\mathrm{C} a^{\prime} \mathrm{B}}=\widehat{\mathrm{CA}} b^{\prime}=c^{\prime} \widehat{\mathrm{AB}}=\pi-(\mathrm{A}+a), \\
& a^{\prime} \widehat{\mathrm{B}} \mathrm{C}=\widehat{\mathrm{A}} \bar{b}^{\prime} \mathrm{C}=\mathrm{A} \widehat{\mathrm{B}} c^{\prime}=\pi-(\mathrm{B}+\beta), \\
& \mathbf{B} \widehat{\mathrm{C}} a^{\prime}=b^{\prime} \widehat{\mathrm{CA}}=\widehat{\mathrm{B} c^{\prime} \mathrm{A}}=\pi-(\mathrm{C}+\gamma),
\end{aligned}
$$

then $\mathrm{A} a^{\prime}, \mathrm{B} b^{\prime}, \mathrm{C} c^{\prime}$ cointersect in a point, which $\mathrm{I}$ shall denote by $\Pi^{\prime}$, whose trilinear coordinates are

$$
\operatorname{cosec} \alpha \sin (\mathbf{A}+\alpha), \operatorname{cosec} \beta \sin (\mathbf{B}+\beta), \operatorname{cosec} \gamma \sin (\mathbf{C}+\gamma) .
$$

The points $I I$ and $I I$ are obviously isogonal conjugates with respect to the triangle $A B C$.

They will be referred to subsequently as the $\Pi$-points of the S-point system of circles whose angles are $\mu, \beta, \gamma$. It will be found that the $\Pi$-points of an $\$$-point system play an analogous part to that played by the Brocard points, which are a particular case of them, in the theory of the Tucker circles.

Other methods of constructing the $\Pi$-points of a system might easily be deduced from the following considerations.

$\Pi a \mathrm{BC}, \Pi \mathrm{A} b \mathrm{C}, \Pi \mathrm{AB} c, \Pi^{\prime} a^{\prime} \mathrm{BC}, \Pi^{\prime} \mathbf{A} b^{\prime} \mathrm{C}, \Pi^{\prime} \mathbf{A B} c^{\prime}$

are evidently quartets of concyclic points.

Hence $\mathrm{B} \widehat{\Pi} \mathrm{C}=\pi-a$, etc., $\Pi \widehat{\mathrm{B} C}+\mathrm{II} \widehat{\mathrm{CB}}=a$, etc.,

$\widehat{\mathrm{B}} \widehat{\Pi^{\prime}} \mathrm{C}=\mathbf{A}+\alpha$, etc., $\Pi^{\prime} \widehat{\mathrm{B}} \mathrm{C}+\Pi^{\prime} \widehat{\mathrm{C}} \mathrm{B}=\pi-(\mathbf{A}+\alpha)$, etc.

6. The pedal circle of the II-points of an S-point system of circles connected with the triangle $\mathrm{ABC}$, is one of the circles of the system.

Demonstration. Let $l, m, n$ (Fig 8 ) be the feet of the perpendiculars from II on BC, CA, AB respectively, and $L, M, N$ the feet of the corresponding perpendiculars from $\Pi^{\prime}$. Then, by a well-known theorem of Steiner's, since $\Pi$ and $\Pi^{\prime}$ are isogonal conjugates $\mathrm{L}, l, \mathrm{M}, m, \mathrm{~N}, n$, lie on a circle. Hence, making the necessary joins and noticing that BLM'N and $C M \Pi^{\prime} L$ are cyclic quadrilateral we have

$$
\widehat{A n M}=\widehat{N} \widehat{L} M=\Pi^{\prime} \widehat{B} A+\Pi^{\prime} \widehat{C A}=\Pi \widehat{B C}+\Pi \widehat{C B}=a .
$$


Similarly it may be shown that $\mathbf{B} \hat{l} \mathrm{~N}=\beta$, and $\widehat{\mathrm{Om} L}=\gamma$. Therefore the circle $\mathrm{L} l \mathrm{M} m \mathrm{~N} n$ belongs to the S-point system whose angles are $a, \beta, \gamma$, i.e., to the system whose $\Pi$-points are $\Pi$ and $\mathrm{II}^{\prime}$.

It will be noticed that the centre of the circle $\mathrm{L} l \mathrm{M} m \mathrm{~N} n$ is the mid-point of III'.

It is evident that an S-point system connected with the triangle is completely determined, if its $\Pi$-points are given.

7. If the pedal triangles of $I I$ and $\Pi^{\prime}$ be rotated round $\Pi$ and $\Pi^{\prime}$ respectively, in opposite directions through equal angles, so as to remain inscribed in $A B C$ and retain their species, they have a common circumcircle which belongs to the S-point system of which $\Pi$ and $\Pi^{\prime}$ are the $\Pi$-points.

Demonstration. Let $\mathrm{Hl}, \Pi m, \Pi n$ (Fig. 8 ) be rotated round $\Pi$ in, say, the positive direction through an angle $\phi$, and in their new positions let them meet the sides of $\mathrm{ABC}$ in $l^{\prime}, m^{\prime}, n^{\prime}$. Similarly let $\Pi^{\prime} L, \Pi^{\prime} M, \Pi^{\prime} N$ be rotated round $\Pi^{\prime}$ in the negative direction through an equal angle $\phi$, meeting in their new positions the sides of $\mathrm{ABC}$ in $\mathrm{L}^{\prime}, \mathrm{M}^{\prime}, \mathrm{N}^{\prime}$. Then, as might easily be proved, the triangles $l m n$ and LMN remain constant in species during the above operation; and $L^{\prime} l^{\prime} \mathbf{M}^{\prime} m^{\prime}, \mathbf{M}^{\prime} m^{\prime} \mathbf{N}^{\prime} n^{\prime}, \mathbf{N}^{\prime} n^{\prime} L^{\prime} l^{\prime}$ are cyclic quartets, whence by Art. $1, \mathrm{~L}^{\prime}, l^{\prime}, \mathrm{M}^{\prime}, m^{\prime}, \mathrm{N}^{\prime}, n^{\prime}$ are concyclic.

Again, making the necessary joins, and denoting the angles IIAB, II'AC by $\theta$, we have

and

$$
\begin{aligned}
& \mathbf{M M}^{\prime}=\Pi^{\prime} \mathbf{M} \tan \phi=\mathbf{A M} \tan \theta \tan \phi, \\
& n n^{\prime}=\Pi n \tan \phi=\mathrm{A} n \tan \theta \tan \phi .
\end{aligned}
$$

Therefore $\mathbf{M}^{\prime} n^{\prime}$ is parallel to $\mathbf{M} n$. Hence $\widehat{A} \widehat{n^{\prime}} \mathbf{M}^{\prime}=u$.

Similarly it may be shown that $\widehat{\mathrm{B}} \widehat{l^{\prime}} \mathrm{N}^{\prime}=\beta$, and $\widehat{\mathrm{C}} \mathrm{n}^{\prime} \mathrm{L}^{\prime}=\gamma$. Therefore the circle $L^{\prime} l^{\prime} \mathbf{M}^{\prime} m^{\prime} \mathrm{N}^{\prime} x^{\prime}$ belongs to the $\mathrm{S}$-point system whose angles are $\alpha, \beta, \gamma$, i.e. to the system whose $\Pi$-points are $\Pi$ and $\Pi^{\prime}$.

It will be noticed that

$$
\widehat{n l m}=\Pi \widehat{\mathrm{BA}}+\Pi \widehat{\mathrm{CA}}=\Pi^{\prime} \mathrm{BC}+\Pi^{\prime} \widehat{\mathrm{CB}}=\pi-(\mathrm{A}+a) \text {, etc., }
$$

and $\widehat{\mathrm{NLM}}=\Pi \widehat{\mathrm{BA}}+\Pi^{\widehat{C A A}}=\Pi \widehat{\mathrm{BC}}+\Pi \widehat{\mathrm{CB}}=a$, etc.

Hence the angles of the variable triangle $\left(l^{\prime} m^{\prime} n^{\prime}\right)$ ussociated with $\Pi$, are equal to the supplements of $\mathrm{A}+\alpha, \mathrm{B}+\beta, \mathrm{C}+\gamma$, while those of the variable triangle $\left(\mathrm{L}^{\prime} \mathrm{M}^{\prime} \mathrm{N}^{\prime}\right)$ associated with $\Pi^{\prime}$, are equal to $a, \beta, \gamma$. 
An interesting particular case not amongst those discussed in Part II. may be referred to here. When $\Pi$ is on the circumcircle of $\mathrm{ABC}, \mathrm{I}^{\prime}$, its isogonal conjugate, is at infinity, and the pedal triangle of $\Pi$ degenerates into the Simson line of $\Pi$. In this case the circles of the S-point system degenerate into a system of linepairs, one line of each pair being the line at infinity and the other lines the Simson lines of $\Pi$ (the lines joining the feet of isoclinals from $\Pi$ to the sides of $A B C$ being included in this designation).

8. The locus of the centres of an S-point system of circles is the straight line which bisects the connector of the $\Pi$-points perpendicularly.

Demonstration. Let $\mathrm{K}, \mathrm{K}^{\prime}$ (Fig. 8) be the centres of the circles $L l M m N n$ and $L^{\prime} l^{\prime} M^{\prime} m^{\prime} \mathbf{N}^{\prime} n^{\prime}$ respectively, and join $K K^{\prime}, K^{\prime} \Pi, K^{\prime} \Pi^{\prime}$. Then $\mathbf{K}$ is the mid-point of $\Pi \Pi^{\prime}$, and the angles $\mathbf{K} \Pi \mathbf{K}^{\prime}, \mathbf{K} \Pi^{\prime} \mathbf{K}^{\prime}$ are obviously each equal to the angle of rotation $\phi$. Therefore KK' bisects $\Pi \Pi^{\prime}$ perpendicularly.

When $\mathrm{L} m, \mathrm{~L}^{\prime} m^{\prime}$ etc., are equally inclined to $\mathrm{BC}, \mathrm{CA}$ and $\mathrm{N} l, \mathrm{~N}^{\prime} l$, etc., to $\mathrm{AB}, \mathrm{BC}$, it is obvious that the perpendicular bisectors of $\mathrm{Lm}, \mathrm{L}^{\prime} \mathrm{m}^{\prime}$, etc., coalesce, as also those of $\mathrm{N} l, \mathrm{~N}^{\prime} l^{\prime}$, etc.; so that in this case the line of centres reduces to a point.

9. The radii of the circles of an S-point system bear a constant ratio to the distances of their centres from either of the $\Pi$-points of the system.

Demonstration. If $\mathbf{K} n, \mathbf{K}^{\prime} n^{\prime}$ (Fig. 8) be joined, it is obvious that the triangles $\Pi K n, \Pi K^{\prime} n^{\prime}$ are similar. Hence $\mathrm{K}^{\prime} n^{\prime} / \mathrm{K} n=\mathrm{K}^{\prime} \Pi / \mathrm{K} \Pi$, which proves the proposition.

From this property it is evident that if we construct a system of coaxal circles having $\Pi$ and $\Pi^{\prime}$ as common points, the circles of the S-point system are simply those of the coaxal system with their radii increased or diminished in a constant ratio, the centres remaining fixed. I propose to call a system of circles arrived at in this manner, by the elongation or shortening in a constant ratio of the radii of a coaxal system whether of the common-point or limitingpoint species, a coaxaloid system of circles. The ratio in which the radii of the kernel coaxal system are increased or diminished will be referred to as the ratio of the resulting coaxaloid system. It is 
evident that coaxaloid systems of circles could be studied altogether apart from their connection with the triangle, and in fact many of their properties can be so obtained, of which the theorems of Arts. 14 to 17 are examples. It is evident also that while every S-point system as previously defined, is a coaxaloid system, it does not follow that every coaxaloid system is an S-point system of a triangle with which its II-points are connected as isogonal conjugates. In fact for every pair of real $\Pi$-points, connected with a triangle as isogonal conjugates, we have an infinite number of coaxaloid systems with different ratios, but only one of these systems with a definite ratio depending on the position of the $\Pi$-points, is a system of six-point circles of the triangle, possessing the property of parallel chords. When the $\Pi$-points are imaginary, i.e. when the coaxaloid systems are derived from a limiting-point instead of a common-point coaxal system, none of the coaxaloid systems are S-point systems. In future an S-point system of circles will be called a coaxaloid system connected with the triangle.

10. $\Pi$ and $\Pi^{\prime}$ being the $\Pi$-points of a coaxaloid system of circles connected with the triangle, we will now consider the connection between the magnitude of the ratio of the system, denoted by $r$, and the position of $I I$ and II'. Let $d$ denote the diameter of the pedal circle of $\Pi$ and $\Pi^{\prime}$. Then $d / \Pi \Pi^{\prime}=r$.

(i) When $\mathrm{II}$ and $\mathrm{II}^{\prime}$ both lie within the triangle, $d>\mathrm{III}^{\prime}$ and $r>1$.

(ii) When $I I$ and $\Pi^{\prime}$ lie on the perimeter of the triangle, in which case one of them coincides with a vertex and the other lies on the opposite side, $d=\Pi \Pi \Pi^{\prime}$, and $r=1$. In this case the coaxaloid system connected with the triangle is an ordinary common-point coaxal system.

(iii) When one of the II-points lies outside the triangle but within the circumcircle, in which case the other lies outside the circumcircle, $d<\Pi \Pi^{\prime}$, and $r<1$.

(iv) When one of the II-points lies on the circumcircle, in which case the other is at infinity (see Art. 8), $d=\Pi \Pi^{\prime}=\infty$, and $r=1$.

(v) When $\Pi$ and $\Pi^{\prime}$ both lie outside the circumcircle, $d>\Pi^{\prime} \Pi^{\prime}$ and $r>1$. 
(vi) When $\Pi$ and $\Pi^{\prime}$ coincide, as they do in the incentre and excentres, $\Pi \Pi \Pi^{\prime}=0$ and $r=\infty$.

(vii) If $\mathrm{K} l$ (Fig. 8) be joined it may easily be proved that $4 \mathrm{~K} l^{2}=\Pi \Pi^{\prime 2}+4 \Pi l . \Pi \Pi^{\prime} \mathrm{L}$. But $\mathrm{K} l$ is a radius of the pedal circle of $\Pi$ and $\Pi^{\prime}$. Hence $r^{2}=1+4 \Pi l$. $\Pi^{\prime} L / \Pi \Pi^{\prime 2}$. This result supplies us with a ready means of calculating the ratio in certain cases.

11. The envelope of a coaxaloid system of circles connected with a triangle $\mathrm{ABC}$ is the conic inscribed in (or escribed to) the triangle, and having the $\Pi$-points of the system as foci.

Demonstration. Let $\mathrm{K}$ be the mid-point of $\Pi \Pi^{\prime}$ (Fig. 9), and TT' the diameter through $\Pi$ and $\mathrm{II}^{\prime}$ of the pedal circle of $\Pi$ and $\Pi^{\prime}$. Let $\mathrm{K}^{\prime}$ be the centre of any other circle of the system, and let this

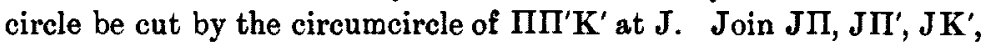
II $K^{\prime}$ and $\Pi^{\prime} K^{\prime}$.

(i) Suppose $r>1$, and consequently KT $>K \Pi$. Then by Ptolemy's theorem

But $\mathbf{K}^{\prime} \mathrm{II}^{\prime}=\mathbf{K}^{\prime} \mathrm{I}$.

Therefore

$$
J \Pi \cdot K^{\prime} \Pi^{\prime}+J \Pi^{\prime} \cdot K^{\prime} \Pi=\Pi \Pi^{\prime} . K^{\prime} J .
$$

$$
\begin{aligned}
\mathrm{J} \Pi & +\mathrm{J}^{\prime}=\Pi \Pi^{\prime} . \mathrm{K}^{\prime} \mathbf{J} / \mathrm{K}^{\prime} \Pi \\
& =\Pi \Pi^{\prime} . \mathrm{KT} / \mathrm{K} \Pi=\mathrm{TT}^{\prime} .
\end{aligned}
$$

Therefore $J$ lies on an ellipse having $\Pi$ and $\Pi^{\prime}$ as foci, and the pedal circle of $\Pi$ and $\Pi^{\prime}$ as auxiliary circle.

Again, since $K^{\prime} \Pi^{\prime}=K^{\prime} \Pi$, JK' bisects the angle $\Pi J \Pi^{\prime}$, and is, consequently, the normal to the ellipse at $J$. Therefore the circle $\mathbf{K}^{\prime}$ touches the ellipse at $J$. Similarly it might be proved to touch the ellipse at the image of $J$ with respect to the line of centres of the coaxaloid system.

Again, since the feet of the perpendiculars from $\Pi$ and $\Pi^{\prime}$ on the sides of $\mathrm{ABC}$, lie on the pedal circle of $\Pi$ and $\mathrm{II}^{\prime}$, i.e. on the auxiliary circle of the ellipse, the sides of $A B C$ are tangents to the ellipse.

It may be noted that when $\Pi$ and $\Pi^{\prime}$ coincide, the ellipse becomes the incircle or one of the excircles.

(ii) When $r<1$, it may be proved in precisely the same way that the envelope is a hyperbola touching the sides of $A B C$ and having $\Pi, \Pi^{\prime}$ as foci and their pedal circle as auxiliary circle. 
(iii) In the particular case when one of the $\Pi$-points lies on the circumcircle, the coaxaloid system degenerates, as we have seen, into the Simson lines of the point, and the other $\Pi$-point lies at intinity. In this case, therefore, the envelope becomes a parabola. We thus obtain the well-known theorem that the envelope of the Simson lines of a point is a parabola escribed to the triangle and having the point as focus.

It is obvious that the foregoing proof establishes the more general theorem that the envelope of a coaxaloid system of circles, without restriction as to connection with the triangle, is a conic having the $\Pi$-points of the system as foci, and the minimum circle of the system as auxiliary circle.

12. The envelope of a coaxaloid system of circles connected with the triangle, touches the sides of the triangle at the points where these are met by the lines joining the S-point to the opposite vertices.

Demonstration. Let $\mathrm{ABC}$ (Fig. 10) be the triangle, and $\mathrm{S}$ any point in its plane. Let $\mathrm{AS}, \mathrm{BS}, \mathrm{CS}$ meet $\mathrm{BC}, \mathrm{CA}, \mathrm{AB}$ in $\mathrm{D}, \mathrm{E}, \mathrm{F}$ respectively. Then by Art 3 (ii) one of the circles of the coaxaloid system connected with the triangle and having $\mathbf{S}$ as $\mathbf{S}$-point, touches $B C$ at $D$. This is sufficient to prove that the envelope of the system also touches $B C$ at $D$. Similarly it might be shown that it touches $\mathrm{CA}$ and $\mathrm{AB}$ at $\mathrm{E}$ and $\mathrm{F}$ respectively.

We are now in a position to find a geometrical solution of the following problem: Given the S-point of a coaxaloid system of circles connected with the triangle $\mathrm{ABC}$, to find the $\Pi$-points, and thus determine the system generally.

Solution. Let X, Y, Z (Fig. 10) be the mid-points of the connectors EF, FD, DE respectively. Then since $A B C$ is circumscribed to the envelope of the system and DEF is inscribed in it, the connectors $\mathrm{AX}, \mathrm{BY}, \mathrm{CZ}$ meet in the centre of the envelope. Let this point be denoted by $K$. It may be noted that $S$ and $K$ coincide when $S$ is the centroid of $A B C$. If now two points $J$ and $J^{\prime}$ be taken on $A K$, on opposite sides of $K$, such that $\mathbf{K J}^{2}=\mathbf{K J}^{\prime 2}=\mathbf{K X} . \mathbf{K A}$, $\mathbf{J} \mathbf{J}^{\prime}$ is obviously the diameter of the envelope conjugate to the chord EF. Being given a chord and the conjugate diameter, we can find the foci of the envelope by any one of a number of well-known 
constructions, i.e., we can find the $\Pi$-points of the system. Then by drawing perpendiculars $\Pi l, \Pi m, \Pi n, \Pi^{\prime} L, \Pi^{\prime} \mathbf{M}, \Pi^{\prime} \mathbf{N}$ from these points to the sides of $A B C$ as in Fig. 8, we determine one circle of the system, and by drawing parallels (or antiparallels) to the connectors $\mathrm{Mn}, \mathrm{N} l, \mathrm{Lm}$ so as to intersect two and two on CS, AS, BS we determine the other circles of the system.

The following particular case of the problem just solved may be noticed: Given a point $S$ in the plane of a triangle, to draw through $\mathbf{S}$ three lines terminated by the sides of the triangle, two and two, such that their extremities shall lie on a circle.

Combining the theorem of the present article with the proposition that every six-point circle connected with the triangle belongs, in general, to four coaxaloid systems connected with the triangle, we obtain the following interesting result. Every six-point circle connected with the triangle has, in general, double contact (real or imaginary) with four conics which touch the sides of the triangle at the points, taken three and three, where the connectors of the vertices with the four S-points meet the opposite sides.

13. Every coaxaloid system of circles having real $\Pi$-points, is an S-point system of an infinite number of triangles, viz., of all the triangles circumscribed to the envelope of the system.

Demonstration. Let $\Pi$ and $\Pi^{\prime}$ be the $\Pi$-points of a coaxaloid system, and let any triangle be circumscribed to its envelope. The minimum circle of the system is the auxiliary circle of the envelope, and therefore, by a well-known property of tangents to a conic, is the pedal circle of $\Pi$ and $\Pi^{\prime}$ with respect to the triangle. Again, by another well-known property of tangents to a conic, $\Pi$ and $\Pi^{\prime}$ are isogonal conjugates with respect to the triangle, and hence are the II-points of an S-point system connected with the triangle. Of this system the pedal circle of $\Pi$ and $\Pi^{\prime}$ with respect to the triangle is one of the circles. Hence the ratio of both systems-the coaxaloid and the S-point system-is the diameter of the pedal circle of $\Pi$ and $\Pi^{\prime}$ over the distance $\Pi \Pi^{\prime}$. Therefore, since both systems have the same II-points and the same ratio, they are identical, which proves the proposition.

The theorem is important as affording a starting-point for the discussion of coaxaloid systems of circles connected with the quadrilateral and general polygon. 
14. The tangent to the minimum circle of a coaxaluid systom (with real II-points) at either extremity of the diameter through II and II' meets every other circle of the system in two points which connect with the centre of the latter through II and $\Pi^{\prime}$.

Demonstration. Let $\mathrm{K}$ (Fig. 9) be the mid-point of III', and, therefore, the centre of the minimum circle, and $K^{\prime}$ the centre of any other circle of the system. Let the tangent to the mininum circle at $\mathbf{T}$, one of the extremities of the diameter through $\Pi$ and $\Pi^{\prime}$, meet the connectors $\Pi K^{\prime}, \Pi^{\prime} K^{\prime}$ in $U$ and $U^{\prime}$ respectively. Then by Euc. VI., 2, $\mathrm{K}^{\prime} \mathrm{U} / \mathrm{K}^{\prime} \Pi=\mathrm{KT} / \mathrm{K} \Pi$. Therefore, by Art. 10, U lies on the circle $K^{\prime}$. Similarly $K^{\prime} U^{\prime} / K^{\prime} \Pi^{\prime}=K T / K I^{\prime}$. Therefore $U^{\prime}$ also lies on the circle $K^{\prime}$.

15. The circles of the coaxal system from which a coaxaloid system is derived, are the circles of similitude of the circles of the coaxaloid system taken in pairs.

Demonstration. Let $\mathrm{K}^{\prime}, \mathrm{K}^{\prime \prime}$ (Fig. 9) be the centres of two circles of the coaxaloid system, $\mathbf{E}$ their external and $\mathrm{I}$ their internal centre of similitude. Then $\mathrm{EK}^{\prime} / \mathbf{E K}^{\prime \prime}=\mathrm{K}^{\prime} \mathrm{T} / \mathbf{I K}^{\prime \prime}$

$$
\begin{aligned}
& =\text { radius of circle } \mathbf{K}^{\prime} / \text { radius of circle } \mathbf{K}^{\prime \prime} \\
& =\Pi \mathbf{K}^{\prime} / \Pi \mathbf{K}^{\prime \prime} .
\end{aligned}
$$

Therefore EI subtends a right angle at $\Pi$, which proves the proposition.

In the proof $\Pi$ and II' are real points, but the same result holds good when they are imaginary.

16. The locus of the extremities of a system of parallel diamaters of a coaxaloid system of circles is a hyperbola.

Demonstration. Through $\mathrm{K}$, the mid-point of $\Pi \Pi^{\prime}$, (Fig. 9) draw uny line $K V$, and from $K^{\prime}$, the centre of any circle of the coaxaloid system, draw $K^{\prime} W$ a radius, parallel to $K V$. Then if $x$ and $y$ are the coordinates of $\mathrm{W}$ with respect to the axes $\mathrm{KV}, \mathrm{KK}^{\prime}$, we have

$$
\begin{aligned}
x^{2} & =\mathrm{K}^{\prime} \mathrm{W}^{2}=\mathrm{K}^{\prime} \Pi^{2} . \mathrm{KT}^{2} / \mathrm{K} \Pi^{2} \\
& =\left(\mathrm{K}^{\prime} \mathrm{K}^{2}+\mathrm{K} \Pi^{2}\right) \mathrm{KT}^{2} / \mathrm{K}^{2} \\
& =\left(y^{2}+\mathrm{K}^{2}\right) \mathrm{KT}^{2} / \mathrm{K}^{2} .
\end{aligned}
$$

Hence the equation of the locus of $\mathrm{W}$ is $x^{2} / \mathrm{KT}^{2}-y^{2} / \mathrm{K} \Pi^{2}=1$, which proves the proposition 
By varying the direction of the parallel diameters we obtain a system of hyperbolas associated with the coaxaloid system. These are obviously concentric, with $K$ as centre, and have a common diameter equal to $\Pi \Pi^{\prime}$ coinciding with the line of centres of the coaxaloid system. Obviously also their diameters conjugate to this common diameter are diameters of the minimum circle of the coaxaloid system; hence the tangents to the hyperbolas at the points where the latter are cut by the minimum circle are perpendicular to $\Pi \Pi^{\prime}$. When the coaxaloid system is coaxal, $\mathrm{KT}=\mathrm{K} \Pi$, and hence the associated hyperbolas are all equilateral. When the diameters of the circles are drawn parallel to $\Pi \Pi$ ', the hyperbola on which their extremities lie has ' $T T^{\prime}$, the diameter through $\Pi$ and $\Pi^{\prime}$ of the minimum circle, as transverse axis, and the common diameter of all the hyperbolas, as conjugate axis. In this case the minimum circle of the coaxaloid system is the auxiliary circle of the hyperbola.

The theorem has been proved for the case when $\Pi$ and $\Pi^{\prime}$ are real, but might easily be extended to the case when they are imaginary.* In the former case the extremities of the parallel diameters of the circles lie on different branches of the hyperbolas, and the common diameter of the latter is ideal ; in the latter case the extremities of the parallel diameters of the circles lie on the same branches of the hyperbolas and the common diameter of the latter is real.

The converse theorem may be stated: Circles described on parallel chords of a hyperbola as diameters form a coaxaloid system, with real $\Pi$-points if the extremities of the chords lie on different branches of the curve, with imaginary II-points if they lie on the same branch. In the particular case when the hyperbola is a linepair, the coaxaloid circles reduce to circles having a common centre of similitude, and the other associated hyperbolas are also line-pairs.

17. The hyperbolas associated with a coaxaloid system have the same envelope as the system.

Demonstration. If $\mathrm{KV}, \mathrm{KK}^{\prime}$ (Fig. 9) be taken as axes, the equation of the hyperbola obtained by drawing diameters of the system parallel to $\mathrm{KV}$ is $x^{2} / a^{2}-y^{2} / c^{2}=1$, where $a=\mathrm{KT}$, and $c=\mathrm{K} \Pi$.

* See nate ut end of paper. 
Transforming to the axes $K T, K K^{\prime}$, we have

$$
x^{2} \sec ^{2} \phi / a^{2}-(y-x \tan \phi)^{2} / c^{2}=1,
$$

where $\phi$ denotes the angle VKT', Rewriting we have

$$
x^{2}\left(a^{2}-c^{2}\right) \tan ^{2} \phi-2 a^{2} x y \tan \phi-c^{2} x^{2}+a^{2} y^{2}+a^{2} c^{2}=0 .
$$

Hence the equation of the envelope of the hyperbolas is

$$
\begin{gathered}
\left(a^{2}-c^{2}\right)\left(a^{2} y^{2}-c^{2} x^{2}+a^{2} c^{2}\right)=a^{4} y^{2}, \\
\text { i.e. } x^{2} / a^{2}+y^{2} /\left(a^{2}-c^{2}\right)=1,
\end{gathered}
$$

which represents the conic having $\Pi, \Pi^{\prime}$ as foci, and 'TT' as transverse axis.

II.

\section{Particular Cases of Coaxaloid Sygtems of Circles connected with the Triangle.}

Some of the more obvious particular cases of coaxaloid systems connected with the triangle $\mathrm{ABC}$ will now be noticed.

18. The Tucker Circles. When the angles $\alpha, \beta, \gamma$ (Art. 4) equal $\mathbf{B}, \mathbf{C}, \mathbf{A}$ (or $\mathbf{C}, \mathbf{A}, \mathbf{B}$ ) respectively, the trilinear coordinates of $\mathbf{S}$ are i.e., $\mathbf{S}$ is the symmedian point.

$$
\sin \mathrm{A}, \sin \mathrm{B}, \sin \mathrm{C} \text {; }
$$

By Art. 5, the trilinear coordinates of the $\Pi$-points are

$$
c / b, a / c, b / a \text { and } b / c, c / a, a / b
$$

i.e., the $\Pi$-points are the Brocard points.

This is the well-known case of the Tucker circles.

The ratio of this system may be calculated in terms of the angles of $\mathrm{ABC}$ as follows: If $\Omega, \Omega^{\prime}$ are the Brocard points, and $\Omega \mathrm{L}, \Omega^{\prime} \mathrm{L}^{\prime}$ be drawn perpendicular to $\mathrm{BC}$, we have, by Art. 11 (vii)

$$
r^{2}=1+4 \Omega L, \Omega^{\prime} L^{\prime} / \Omega \Omega^{\prime 2} \text {. }
$$

Hence, using the well-known results,

$$
\begin{gathered}
\Omega \mathrm{L} . \Omega^{\prime} \mathbf{L}^{\prime}=4 \mathrm{R}^{2} \sin ^{4} \omega, \\
\Omega \Omega^{\prime 2}=4 \mathrm{R}^{2} \sin ^{2} \omega\left(1-4 \sin ^{2} \omega\right),
\end{gathered}
$$

and

$$
\operatorname{cosec}^{2} \omega=\operatorname{cosec}^{2} \mathbf{A}+\operatorname{cosec}^{2} \mathbf{B}+\operatorname{cosec}^{2} \mathrm{C} \text {, }
$$

where $R$ is the radius of the circumcircle, and $\omega$ the Brocard angle, we have

$$
\begin{aligned}
r^{2} & =\left(1-3 \sin ^{2} \omega\right) /\left(I-4 \sin ^{2} \omega\right) \\
& \neq \Sigma \cot ^{2} \mathbf{A} /\left(\Sigma \cot ^{2} \mathbf{A}-1\right) .
\end{aligned}
$$


19. A System derived from the Nine-Point Circle.

If $\mathrm{A}^{\prime}, \mathrm{B}^{\prime}, \mathrm{C}^{\prime}$ are the mid-points of $\mathrm{BC}, \mathrm{CA}, \mathrm{AB}$, and $\mathrm{D}, \mathrm{E}, \mathrm{F}$ the feet of the altitudes on these sides respectively, then the connectors $\mathrm{FB}^{\prime}, \mathrm{DC}^{\prime}, \mathrm{EA}^{\prime}$ are the directive chords of one of the four systems derivable from the nine-point circle.

Since

$\widehat{A} \widehat{F B}^{\prime}=A, \widehat{B D C}=B$, and $\widehat{C E A^{\prime}}=C$,

the angles of the system are $A, B, C$ (or $\pi-2 A, \pi-2 B, \pi-2 C$ ). Hence, by Art. 4, the trilinear coordinates of $S$ are

$$
\sec \mathrm{A} / a^{2}, \sec \mathrm{B} / b^{2}, \sec \mathrm{C} / c^{2} ;
$$

i.e., $\mathrm{S}$ is the isotomic conjugate of the circumcentre. Thus the other circles of the system are obtained by drawing transversals $\mathrm{M} n, \mathrm{~N} l, \mathrm{Lm}$, terminated by the sides as in Fig. 6, so as to intersect two and two on CS, AS, BS, where $S$ is the isotomic conjugate of the circumcentre, and so as to make with the sides of $\mathrm{ABC}$ three isosceles triangles whose vertices are $\mathrm{M}, \mathrm{N}, \mathrm{L}$ (or $m, n, l$ ) respectively.

By Art. 5 the trilinear coordinates of the $\Pi$-points are $\cos \mathrm{A}, \cos \mathrm{B}, \cos \mathrm{C}$ and $\sec \mathrm{A}, \sec \mathrm{B}, \sec \mathrm{C}$;

i.e., the $\Pi$-points are the circumcentre, $\mathrm{O}$, and the orthocentre, $\mathrm{H}$. Thus the line of centres of the system is the perpendicular bisector of $\mathrm{OH}$, and the nine-point circle, being the pedal circle of $\mathrm{O}$ and $\mathrm{H}$, is the minimum circle of the system.

By Art. 10 (viii), and the well-known relations,

and

$$
\mathrm{OA}^{\prime}=\mathrm{R} \cos \mathrm{A}, \mathrm{HD}=2 \mathrm{R} \cos \mathrm{B} \cos \mathrm{C},
$$

we have

$$
\begin{aligned}
\mathrm{OH}^{2} & =\mathrm{R}^{2}(1-8 \cos \mathrm{A} \cos \mathrm{B} \cos \mathrm{C}), \\
r^{2} & =1 /(1-8 \cos \mathrm{A} \cos \mathrm{B} \cos C) .
\end{aligned}
$$

It may be noticed that the following theorem is involved in the foregoing: The connector of $\mathrm{A}$ with the isotomic conjugate of $\mathrm{O}$ contains the points of intersection of the pairs $\mathbf{D C}^{\prime}, \mathbf{E A}^{\prime}$ and $\mathrm{FA}^{\prime}, \mathrm{DB}^{\prime}$; with similar theorems for the vertices $\mathrm{B}$ and $\mathrm{C}$.

20. Other Systems derived from the Nine-Point Circle.

Take $B^{\prime} F, C^{\prime} A^{\prime}, D E$ (notation of preceding article) as directive chords. Since $\widehat{A F B^{\prime}}=A, \widehat{B A^{\prime} C^{\prime}}=C$, and $\widehat{C E D}=B$, the angles of the resulting system are $A, C, B$ (or $\pi-2 A, A, A$ ). Hence by Art. 4 the trilinear coordinates of $S$ are

$$
\operatorname{cosec} 2 \mathrm{~A}, \operatorname{cosec} \mathrm{C}, \operatorname{cosec} \mathrm{B} \text {, }
$$


by which it at once appears that AS is the symmedian through $A$, and the following construction, independent of the nine-point circle, is suggested. On CA, AB describe isosceles triangles $\mathrm{VCA}, \mathrm{WAB}$ having their base angles equal to $A$. Then $B V, C W$ and the symmedian through $A$ cointersect in $\mathrm{S}$.

By Art. 5 the trilinear coordinates of $\Pi$ are $\sin \mathrm{A} \operatorname{cosec} 2 \mathrm{~A}, \sin \mathrm{C} \operatorname{cosec} \mathrm{A}, \sin \mathrm{B} \operatorname{cosec} \mathrm{A}$,

from which it is evident that $\Pi$ lies on the median through $A$, and that, consequently, $\Pi^{\prime}$ lies on the corresponding synmedian, and, therefore on AS. Since B $\widehat{\Pi M O}=\pi-A$ (Art. 5), and $B \Pi^{\prime} D=2 A$, the median through $\mathrm{A}$ meets the circumcircle of $\mathrm{BHC}$ in II, and the symmedian through A meets the circumcircle of BOC in $\Pi^{\prime}$. This suggests a construction for finding $\Pi$ and $\Pi^{\prime}$. The line of centres, which, it may be noted, passes through the nine-point centre, may be found by drawing the perpendicular bisector of the distance between $I I$ and $I^{\prime}$, or it may be found by the following construction. Let $\mathrm{K}$ be the symmedian point of $\mathrm{ABC}$, and at $\mathrm{L}$ where $\mathrm{AK}$ meets $B C$ draw a perpendicular to $B C$ meeting $O K$ in $T$. Then the connector of $T$ with the nine-point centre is the line of centres. This may be proved as follows. Through $\mathrm{L}$ draw $\mathrm{LM}, \mathrm{Lm}$ to meet $\mathrm{CA}$, and $\mathrm{LN}, \mathrm{Ln}$ to meet $\mathrm{AB}$, so that $\mathrm{Lm}$ shall be parallel to $\mathrm{AB}$ and LM antiparallel to it with respect to $\mathrm{C}$, and that $\mathrm{LN}$ shall be parallel to $\mathrm{CA}$, and $\mathrm{L} n$ antiparallel to it with respect to $\mathrm{B}$. Then by Art. 3 (ii) since $\widehat{B L N}=C$, and $\widehat{C M L}=\mathrm{B}$, and $\mathrm{L} n, \mathrm{~L} m$ are the antiparallels of $\mathrm{LN}, \mathrm{LM}$ respectively, the points $\mathrm{L}, \mathrm{M}, m, \mathrm{~N}, n$ lie on a circle of the system which touches $B C$ at $L$, and whose centre, therefore lies on LT. But since AL is a symmedian, and $\widehat{B L N}=\mathrm{C}$, and $\widehat{\mathrm{CmI}}=\mathrm{A}$, and $\mathrm{Ln}, \mathrm{LM}$ are the antiparallels of $\mathrm{LN}$ and $\mathrm{L} m$ respectively, this circle is also a Tucker circle, and, therefore, by a well-known theorem relating to Tucker circles, its centre lies on $\mathrm{OK}$. Therefore $\mathrm{T}$ is the centre of the circle, and since the nine-point circle is another circle of the system, the statement is pro"ed.

Two other analogous systems may be derived from the nine-point circle by taking
(i) $\mathbf{E F}, \mathrm{C}^{\prime} \mathrm{D}, \mathrm{A}^{\prime} \mathrm{B}^{\prime}$ and
(ii) $\mathrm{B}^{\prime} \mathrm{C}^{\prime}, \mathrm{FD}, \mathrm{A}^{\prime} \mathrm{E}$

as directive chords.

The following theorem is easily deducible from the foregoing: 
The point of intersection of $\mathrm{C}^{\prime} \mathrm{A}^{\prime}$ and $\mathrm{DE}$, and also the point of intersection of the antiparallel pair $D F$ and $A^{\prime} B^{\prime}$ lie on the symmedian $\mathrm{AK}$; with similar statements for $\mathrm{BK}$ and $\mathrm{CK}$.

\section{The Three Rectangular Systems.}

The circumcircles of all the rectangles inscribed in $\mathrm{ABC}$ so as to have one of their sides lying on a side of the triangle, form a coaxaloid system connected with the triangle.

Demonstration. Let $l \mathrm{~L} m \mathrm{~N}$ (Fig. 11) be any rectangle inscribed in $\mathrm{ABC}$, having $l \mathrm{~L}$ lying on $\mathrm{BC}, m$ on $\mathrm{CA}$, and $\mathrm{N}$ on $\mathrm{AB}$; and let the circumcircle of the rectangle meet $C A$ again in $M$, and $A B$ again in $n$. Then the connector $M n$ is antiparallel to $m \mathrm{~N}$, and therefore to $\mathrm{BC}$, with respect to $\mathrm{A}$. Therefore the chords $\mathrm{M} n, \mathrm{~N} l, \mathrm{~L} m$ are constant in direction, which proves the proposition.

The system thus determined may be called the $A$ rectangular system. Two other analogous systems are obtained by taking the rectangles so as to have a side coinciding (i) with $\mathrm{CA}$ and (ii) with $A B$. These may be called the $B$ and $C$ rectangular systems respectively.

Since, as is well-known, the six points in which the Cosine circle meets the sides of the triangle can be joined so as to form three rectangles having each a side coincident with a side of the triangle, the Cosine circle belongs to each of the rectangular systems. Hence the three lines of centres of the system cointersect in the centre of the Cosine circle, i.e., in the symmedian point.

The angles of the $A$ system are obviously

$$
\text { C, } \left.\frac{\pi}{2}, \frac{\pi}{2}-\mathrm{C} \text { (or B, } \frac{\pi}{2}-\mathrm{B}, \frac{\pi}{2}\right) \text {. }
$$

Hence, by Art. 4, the trilinear coordinates of the S-point of this system are

$$
\operatorname{cosec} \mathrm{B} \operatorname{cosec} \mathrm{C} \text {, secB, secC, }
$$

by which it is evident that $\mathrm{S}$ lies on the altitude $\mathrm{AD}$, and the following construction is suggested: From $D$, the foot of the altitude $A D$, draw perpendiculars to $\mathrm{CA}$ and $\mathrm{AB}$ meeting the parallel to $B C$ through $A$ in $V$ and $W$ respectively; then $\mathrm{AD}, \mathrm{BV}, \mathrm{CW}$ cointersect in $\mathrm{S}$.

The line of centres can be readily determined without first 
finding the II-points. The side $\mathrm{BC}$ and the altitude $\mathrm{AD}$ are degenerate forms of the rectangles which give rise to the $\mathrm{A}$ system, and, therefore, the line of centres of the system is the line joining the mid-points of $B C$ and $A D$.

To solve the problem of finding the M-points of the A system, let $l \mathrm{LmN}$ (Fig. 11) be the rectangle inscribed in the minimum circle of the system. From $l$ draw a perpendicular to $C A$ meeting $C A$ in $M$ and $L m$ in $\Pi^{\prime}$; and from $L$ draw a perpendicular to $A B$ meeting $\mathrm{AB}$ in $n$ and $\mathrm{N} l$ in $\Pi$. Then it is obvious that $\mathrm{M}$ and $n$ are points on the circumcircle of $l \mathrm{Lm} \mathrm{N}$, and that $\Pi$ and $\Pi^{\prime}$, being the points of concurrence of perpendiculars to the sides at the points where these are cut by the minimum circle, are the II-points. Now $\Pi l / l \mathrm{~L}=\cot B$, and $\Pi^{\prime} \mathrm{L} / l \mathrm{~L}=\cot C$. But since $\Pi^{\prime} N$ and $L n$ are perpendicular to $A B, \Pi^{\prime} \mathbf{L}=$ IIN.

Therefore $\quad \mathrm{N} l / l \mathrm{~L}=\cot \mathrm{B}+\cot \mathrm{C}=\mathrm{BC} / \mathrm{AD}$.

This result determines the species of the réctangle $l \mathrm{~L} m \mathrm{~N}$, which can, consequently, be easily constructed. The $\Pi$-points are then obtained by drawing $l \mathrm{M}, \mathrm{L} n$ perpendicular to $\mathrm{CA}, \mathrm{AB}$ respectively as above.

To determine the ratio of the $A$ system we have

$$
\text { and } \begin{aligned}
\Pi l . \Pi^{\prime} L & =l \mathrm{~L}^{2} \cot B \cot C, \\
\Pi \Pi^{\prime 2} & =l \mathrm{~L}^{2}+\left(\mathrm{N} l-2 \Pi^{\prime} \mathrm{L}\right)^{2} \\
& =l \mathrm{~L}^{2}\left\{l+(\cot \mathrm{B}-\cot \mathrm{C})^{2}\right\} .
\end{aligned}
$$

Hence, by Art. 11 (vii) we obtain

$$
r^{2}=\frac{1+(\cot B+\cot C)^{2}}{1+(\cot B-\cot C)^{2}}
$$

The following is given as an example of the numerous theorems dealing with collinearity and concurrency, which arise in connection with these systems. If $l \mathrm{LM} n, m \mathrm{MN} l, n N \mathrm{~L} m$ are the three inscribed rectangles of the Cosine circle, $l \mathrm{~L}, m \mathrm{M}, n \mathrm{~N}$ coinciding with $\mathrm{BC}, \mathrm{CA}, \mathrm{AB}$ respectively, and $\mathrm{AD}, \mathrm{BE}, \mathrm{CF}$ the altitudes, the following are triads of concurrent lines,

$$
\mathrm{AD}, \mathrm{NL}, l m ; \mathrm{BE}, \mathrm{LM}, m n ; \mathrm{CF}, \mathrm{MN}, n l \text {. }
$$

This follows at once from the fact that pairs of directive chords of the rectangular systems intersect on the altitudes. 


\section{The Four Concentric Systems.}

When to $a, \beta, \gamma$ are assigned the values

$$
\frac{1}{2}(B+C), \frac{1}{2}(C+A), \quad \frac{1}{2}(A+B)
$$

respectively, i.e., when the directive chords $\mathrm{M} n, \mathrm{Nl}, \mathrm{Lm}$ (Fig. 12) make with the sides of $\mathrm{ABC}$ three isosceles triangles whose vertical angles are the interior angles $A, B, C$ respectively, the trilinear coordinates of $S$ are found by Art. 5 (i) to be

$$
\sec ^{2} \frac{A}{2}, \quad \sec ^{2} \frac{B}{2}, \quad \sec ^{2} \frac{C}{2} \text {; }
$$

i.e., $\mathrm{S}$ is the point of concurrence of the lines joining the vertices of $\mathrm{ABC}$ to the points where the incircle touches the opposite sides (Gergonne point).

Since the directive chords in this case are isoclinal to the sides of the triangle taken in pairs, it follows from the last paragraph of Article 8 that the circles of the system are concentric.

The incircle evidently belongs to this system, for if $\mathrm{X}, \mathrm{Y}, \mathrm{Z}$ (Fig. 12) are the points where this circle touches BC, CA, AB respectively, the chords $\mathrm{YZ}, \mathrm{ZX}, \mathrm{XY}$ are obviously parallel to the directive chords of the system. It follows that the incentre is the common centre of the system.

By Art. 5, or otherwise, the $\Pi$-points of the system are found to coincide in the incentre. Hence the incircle besides being the minimum circle is the (ideal) envelope of the system.

It may be noticed also that $a$ and $\pi-(\mathrm{A}+a)$ are in this case equal, and so for the other pairs, and that, consequently, the two variable inscribed triangles of constant species which by their rotation (round the incentre) determine the system, are similar, their angles opposite the vertices $A, B, C$ being equal to $\frac{1}{2}(B+C), \frac{1}{2}(C+A), \frac{1}{2}(A+B)$ respectively.

By Art. 10 (vii), $\quad r=\infty$.

The following particulars may be noted with respect to the figure of the present article (Fig. 12)

(i) $m \mathrm{~N}, n \mathrm{~L}, l \mathrm{M}$ are both parallel and antiparallel (with respect to $\mathrm{A}, \mathrm{B}, \mathrm{C})$ to $\mathrm{M} n, \mathrm{~N} l, \mathrm{~L} m$ respectively.

(ii) $\mathrm{L} l=\mathrm{M} m=\mathrm{N} n$, and $\mathrm{X}, \mathrm{Y}, \mathrm{Z}$ are their mid-points.

(iii) If $\mathrm{P}, \mathrm{Q}, \mathrm{R}$ are the points on $\mathrm{AS}, \mathrm{BS}, \mathrm{CS}$ respectively, 
where $\mathrm{M} n, \mathrm{~N} l, \mathrm{~L} m$ intersect, and $p, q, r$ the points on $\mathrm{AS}, \mathrm{BS}, \mathrm{CS}$ where $m \mathrm{~N}, n \mathrm{~L}, l \mathrm{M}$ intersect,

$$
\begin{aligned}
& \widehat{\mathbf{P Q}}=\widehat{r p q}=\frac{1}{2}(\mathrm{~B}+\mathrm{C}), \\
& \widehat{\mathrm{PQR}}=\widehat{p q r}=\frac{1}{2}(\mathrm{C}+\mathrm{A}), \\
& \widehat{\mathrm{RP}}=\widehat{q r} p=\frac{1}{2}(\mathrm{~A}+\mathrm{B}) .
\end{aligned}
$$

Hence $\mathrm{Ll}, \mathrm{M} m, \mathrm{~N} n$ are antiparallel to $\mathrm{QR}, \mathrm{RP}, \mathrm{PQ}$ with respect to the angles $\mathrm{RPQ}, \mathrm{PQR}, \mathrm{QRP}$ respectively, and to $q r, r p, p q$ with respect to the angles $r p q, p q r, q r p$ respectively. They are also antiparallel to $\mathbf{Y Z}, \mathbf{Z X}, \mathbf{X Y}$ with respect to the angles ZXY, XYZ, YZX respectively.

(iv) $\mathrm{S}$ is the symmedian point of the triangles $\mathrm{PQR}, p q r, \mathrm{XYZ}$.

(v) The circle $\mathrm{L} l \mathrm{M} m \mathrm{~m} n$ is a Tucker circle of the triangles $\mathrm{PQR}, p q r, \mathrm{XYZ}$.

(vi) Hence, if $I$ be the incentre of $A B C, J$ the circumcentre of $\mathrm{PQR}$ and $\mathrm{J}^{\prime}$ the circumcentre of $p q r$, then $\mathrm{I}, \mathrm{S}, \mathrm{J}, \mathrm{J}^{\prime}$ are collinear, and $\mathrm{I}$ is the mid-point of $\mathbf{J J}^{\prime}$.

By taking $S$ as the point of concurrence of the lines joining $A, B, C$ to the points where the excircle opposite to $A$ touches the opposite sides, and drawing $\mathrm{Mn}, \mathrm{Nl}, \mathrm{Lm}$ so as to make with the sides of $\mathrm{ABC}$ three isosceles triangles whose vertical angles are the interior angle at $A$ and the exterior angles at $B$ and $C$, we obtain an analogous system concentric with the excircle and including it. An analogous system exists in connection with each of the other excircles.

\section{Systems connected with the Isogonic Centres.}

When $a=\beta=\gamma=60^{\circ}$ (or $\alpha=120^{\circ}-\mathrm{A}, \beta=120^{\circ}-\mathrm{B}, \gamma=120^{\circ}-\mathrm{C}$ ), the trilinear coordinates of $S$ are found by Art. 4 to be

$$
\operatorname{cosec}\left(60^{\circ}+\mathrm{A}\right), \operatorname{cosec}\left(60^{\circ}+\mathrm{B}\right), \operatorname{cosec}\left(60^{\circ}+\mathrm{C}\right) \text {. }
$$

Hence $S$ is the point of concurrence of the lines joining the vertices of $\mathrm{ABC}$ to the vertices of equilateral triangles deseribed externally on the opposite sides, i.e., it is one of the isogonic centres of ABC.

Using Art. 5 we find that, in the case of this system, $\Pi$ coincides with the isogonic centre. Hence the line of centres of the system is the perpendicular bisector of the distance between the isogonic centre and its isogonal conjugate. 
It follows from Art. 7 that the circles of this system are the circumcircles of a variable equilateral triangle inscribed in $\mathrm{ABC}$.

When two of the angles $\alpha, \beta, \gamma$ are taken each equal to $120^{\circ}$, and the remaining angle to $-60^{\circ}$, an analogous system is obtained for which the trilinear coordinates of both $S$ and $\Pi$ are found to be

$$
\operatorname{cosec}\left(60^{\circ}-\mathrm{A}\right), \operatorname{cosec}\left(60^{\circ}-\mathrm{B}\right), \operatorname{cosec}\left(60^{\circ}-\mathrm{C}\right) \text {. }
$$

Thus $S$ and $\Pi$ coincide in the point of concurrence of the lines joining the vertices of $\mathrm{ABC}$ to the vertices of equilateral triangles described internally on the opposite sides, i.e., in the other isogonic centre of $\mathrm{ABC}$. In this case the directive chords are inclined at angles of $60^{\circ}$ to the corresponding chords in the previous case.

\section{Coaxal Systems.}

It has been remarked in Art. 10 (ii) that a coaxaloid system connected with the triangle reduces to a coaxal system, when the II-points lie on the perimeter of the triangle. In illustration the cases which arise in connection with the circle described on a side as diameter may be referred to.

Let $\mathrm{AD}, \mathrm{BE}, \mathrm{CF}$ be the three altitudes of $\mathrm{ABC}$, and consider the circle described on $\mathrm{BC}$ as diameter. The four systems to which this circle belongs may be obtained by taking the following sets of directive chords,

(i) $\mathrm{CB}, \mathrm{FC}, \mathrm{BE}$, which give $\alpha=\mathrm{B}, \beta=\frac{\pi}{2}-\mathrm{B}, \gamma=\frac{\pi}{2}$,

(ii) $\mathrm{CF}, \mathrm{BC}, \mathrm{BE}$, which give $a=\frac{\pi}{2}, \beta=0, \gamma=\frac{\pi}{2}$,

(iii) $\mathrm{EB}, \mathrm{FC}, \mathrm{CB}$, which give $\alpha=\frac{\pi}{2}-\mathrm{A}, \beta=\frac{\pi}{2}-\mathrm{B}, \gamma=\pi-\mathrm{C}$,

(iv) $\mathrm{EF}, \mathrm{BC}, \mathrm{CB}$ which give $\alpha=\mathrm{C}, \beta=0, \gamma=\pi-\mathrm{C}$.

In the first case we have the A-rectangular system (Art. 21), and in the second, third, and fourth cases we have the cosxal systems which have $\mathrm{BE}, \mathrm{CF}$ and $\mathrm{BC}$, respectively, as common chords.

\section{III.}

\section{Additionat Theorems.}

25. The envelope of the polars of a point with respect to the circles of a coaxaloid system is a parabola. 
Demonstration. The equation of any circle of the system referred to $\Pi^{\prime} \Pi^{\prime}$ and $\mathrm{KK}^{\prime}$ as axes (Fig. 9) is

$$
x^{2}+(y-c \tan \phi)^{2}=a^{2} \sec ^{2} \phi
$$

where $\mathrm{KT}^{\prime}=a, K \Pi^{\prime}=c$, and $\phi$ denotes the angle subtended at $\Pi^{\prime}$ by the distance of the centre of the circle from $\mathbf{K}$.

The equation of the polar of a point $x^{\prime}, y^{\prime}$ with respect to the circle is

$$
\left(c^{2}-a^{2}\right) \tan ^{2} \phi-c\left(y+y^{\prime}\right) \tan \phi+x x^{\prime}+y y^{\prime}-a^{2}=0 .
$$

The equation of the envelope of this, when $\phi$ varies, is

$$
4\left(c^{2}-a^{2}\right)\left(x x^{\prime}+y y^{\prime}-a^{2}\right)=c^{2}\left(y+y^{\prime}\right)^{2},
$$

which represents a parabola.

When the system is coaxal, $c=a$, and the parabola, as is well known, reduces to a point.

26. The locus of the poles of a line with respect to the circles of a coaxaloid system is, in general, a hyperbola, but when the line is parallel to the line of centres it is a parabola.

Demonstration. Let the line meet the line of centres at $O$ (Fig. 9) and make with it an angle $a$. Let $K^{\prime} M$ be the perpendicular to the line from $K^{\prime}$, the centre of a circle of the system, and let $P$ be the pole of the line with respect to the circle $\mathrm{K}$ '.

Let $\quad \mathrm{K} \Pi=c, \mathrm{OK}=d$, and $r=$ the ratio of the system.

OK is taken as the axis of $x$, and the line through $O$ parallel to the fixed direction $K^{\prime} M$ as the axis of $y$.

Then $K^{\prime} P \cdot K^{\prime} M=\left(\text { radius of circle } K^{\prime}\right)^{2}=r^{2} \cdot K^{\prime} \Pi^{2}=r^{2}\left(c^{2}+K^{\prime} K^{2}\right)$.

$$
=r^{2}\left(c^{2}+\overline{\mathrm{K}^{\prime} \mathrm{O}-d^{2}}\right) \text {. }
$$

And $\mathbf{K}^{\prime} \mathbf{M}=\mathbf{K}^{\prime} \mathrm{O} \cdot \sin \alpha$.

Hence we obtain as the equation of the locus of $P$,

$$
\begin{gathered}
\left.y x \sin \alpha=r^{2}\left(c^{2}+\overline{x-d}\right)^{2}\right), \\
\text { i.e., } \quad r^{2} x^{2}-x y \sin \alpha-2 r^{2} d x+r^{2}\left(c^{2}+d^{2}\right)=0,
\end{gathered}
$$

which represents a hyperbola, except when $\sin \alpha=0$, when it represents a parabola.

This theorem holds also when the circles are coaxal.

27. The envelope of the radical axes of any circle and the circles of a coaxaloid system is a parabola. 
Demonstration. As in Art. 25 the equation of any circle of the coaxaloid system referred to $\Pi \Pi^{\prime}$ and $\mathrm{KK}^{\prime}$ as axes (Fig. 9) is

$$
x^{2}+(y-\operatorname{ctan} \phi)^{2}=a^{2} \sec ^{2} \phi \quad \text { - . . . }
$$

Let the equation of a circle not belonging to the system be

$$
(x-p)^{2}+(y-q)^{2}=r^{2} \quad-\quad \cdot \quad-
$$

Then the equation of the radical axis of (1) and (2) is

$$
\begin{aligned}
& \left(c^{2}-a^{2}\right) \tan ^{2} \phi-2 c y \tan \phi \\
& \quad+2 p x+2 q y+r^{2}-p^{2}-q^{2}-a^{2}=0 .
\end{aligned}
$$

Therefere the equation of the envelope is

$$
c^{2} y^{2}=\left(c^{2}-a^{2}\right)\left(2 p x+2 q y+r^{2}-p^{2}-q^{2}-a^{2}\right),
$$

which represents a parabola.

When the system is coaxal, $c=a$, and the parabola, as is well known, reduces to a point.

28. Suppose that CA in Fig. 8 is made to move, in contact with the envelope of the system, until it becomes parallel to BC.

Then, in this case, the angles $I^{\prime} \Pi^{\prime} M^{\prime}$ and $l^{\prime} \Pi m^{\prime}$ which are supplementary to the angle $\mathrm{C}$, become each equal to two right angles, so that $L^{\prime} M^{\prime}, l^{\prime} m^{\prime}$ pass through $\Pi^{\prime}$ and $\Pi$ respectively.

Again, since in the general case, $\Pi^{\prime} L^{\prime}, \Pi l^{\prime}$ are equally inclined to $\mathrm{BC}, \mathrm{L}^{\prime} \mathrm{M}^{\prime}, l^{\prime} m^{\prime}$ are equally inclined to $\mathrm{BC}$ and $\mathrm{CA}$, and therefore are equal. Hence the segments, known to be of constant form, cut off from the variable circle $L^{\prime} l^{\prime} M^{\prime} m^{\prime}$ by $L^{\prime} M^{\prime}$ and $l^{\prime} m^{\prime}$ are, in this case, equal for each position of the circle.

Also when the circle $L^{\prime} l^{\prime} \mathbf{M}^{\prime} m^{\prime}$ is the auxiliary circle of the envelope, $L^{\prime} M^{\prime}, l^{\prime} m^{\prime}$ are perpendicular to $B C$ and $C A$. Therefore $L^{\prime} m^{\prime}=l^{\prime} \mathbf{M}^{\prime}=$ diameter of the auxiliary circle

$$
=\text { focal axis of the envelope. }
$$

But since $\mathrm{BC}, \mathrm{CA}$ are parallel, $\mathrm{L}^{\prime} m^{\prime}, l^{\prime} \mathrm{M}^{\prime}$, already known to be fixed in direction, are also constant in length for all positions of the circle $L^{\prime} l^{\prime} M^{\prime} m^{\prime}$, and are therefore each equal to the focal axis of the envelope. From this it follows that they are equally inclined to $\mathrm{BC}$ and $\mathrm{CA}$. By making $\mathrm{L}^{\prime}$ and $l^{\prime}$ coincide at $\mathrm{D}$, the point where $\mathrm{BC}$ touches the envelope, we see that the ixed directions of $\mathrm{L}^{\prime} \mathrm{m}^{\prime}, l^{\prime} \mathrm{M}^{\prime}$ are parallel to $\Pi D, \Pi^{\prime} D$ respectively.

The foregoing may be summed up in the following theorem, of which that of Art. 15 is a particular case. 
If two fixed parallel tangents to the envelope of a coaxaloid system whose $\Pi$-points are $\Pi$ and $\Pi^{\prime}$, meet a variable circle of the system in the points $\mathrm{L}, l$ and $\mathrm{M}, m$, then

(i) Two of the connectors of these four points, say LM and $l m$, pass through $\Pi^{\prime}$ and $\Pi$ respectively, and LM, $l m$ are equally inclined to $\mathrm{L} l$ and $\mathrm{M} m$, and cut off two pairs of equal segments of constant form from the circle; and

(ii) Lm, lM are constant in length being each equal to the focal axis of the envelope, and are fixed in direction being parallel to $\Pi D, \Pi^{\prime} E$ and $\Pi^{\prime} D, \Pi E$ respectively, where $D$ and $E$ are the points of contact of $\mathrm{L} l$ and $\mathrm{Mm}$ with the envelope.

It might be easily proved also that

$\mathrm{LD} / \mathrm{D} l=l \Pi / \mathrm{II} m=\mathrm{ME} / \mathbf{E} m=\mathbf{M} \Pi^{\prime} / \Pi^{\prime} \mathrm{L}=\Pi \Pi^{\prime} \mathrm{D} / \mathrm{D} \Pi=\Pi \mathbf{E} / \mathbf{E} \Pi^{\prime}$.

The following theorem is deducible from the foregoing as a particular case.

If a circle have double contact with a conic whose foci are $I I$ and $\Pi^{\prime}$, and lines be drawn from $L$, one of the points of contact, through $\Pi$ and $\Pi^{\prime}$ meeting the circle in $M$ and $m$ respectively, then $\mathrm{Mm}$ is the tangent to the conic parallel to the tangent at $\mathrm{L}$, and $\mathrm{LM}=\mathrm{Lm}=$ the focal axis of the conic.

Another interesting particular case might be obtained by supposing that $\mathrm{L} l$ and $\mathrm{M} m$ coincide on an asymptote to the envelope.

29. If LMN (Fig. 13) be a variable triangle of constant species inscribed in the triangle $A B C$, not only the circumcircle of LMN, but any circle invariably connected with it (such as the incircle, Taylor circle, Brocard circle, etc.) forms, in its various positions, a coaxaloid system.

Demonstration. Let $\Pi$ be the centre of similitude of the triangle LMN in its various positions. Draw through $I I$ three lines making constant angles with MN, NL, LM respectively, and meeting the circle in $l, m, n$ and the sides $\mathrm{BC}, \mathrm{CA}, \mathrm{AB}$ in $l^{\prime}, m^{\prime}, n^{\prime}$ respectively.

Then the ratios $\Pi L / \Pi l$, etc., are constant, and also the ratios $\Pi L / \Pi l^{\prime}$, etc. Therefore the ratios $\Pi l / \Pi l^{\prime}$, etc., are constant. Hence $l m n$ is a triangle of constant species, and $l, m, n$ move on straight lines parallel to $\mathbf{B C}, \mathbf{C A}, \mathbf{A B}$ respectively. That is the circle is the circumcircle of a variable triangle of constant species inscribed in a fixed triangle, and, therefore, by Art. 7, forms a coaxaloid system. 
If the circle passes through $\Pi$ the system is coaxal ; if LMN is similar to the orthocentric triangle of $\mathrm{ABC}$, and the circle the incircle of LMN, the system is concentric.

30. If a system of directly similar triangles have the same centre of similitude when considered in pairs, the circle passing through the points where the sides of a variable triangle of the system meet the homologous sides of a fixed triangle of the system, forms, in its various positions, a coaxaloid system having the centre of similitude as one of its $\Pi$-points.

Demonstration. In the figure (Fig. 14) $\Pi$ is the common centre of similitude, $A B C$ the fixed triangle, $A^{\prime} B^{\prime} C^{\prime}$ the variable triangle similar to it, $\mathrm{L}, \mathrm{M}, \mathrm{N}$ the points where $\mathrm{BC}, \mathrm{CA}, \mathrm{AB}$ are intersected by the homologous sides of $\mathrm{A}^{\prime} \mathrm{B}^{\prime} \mathrm{C}^{\prime}$ respectively, and $l, m, n$ the feet of the perpendiculars from $\Pi$ on $\mathrm{BC}, \mathrm{CA}, \mathrm{AB}$.

Then, since $\Pi A B, \Pi A^{\prime} B^{\prime}$ are equal angles, $\Pi, A, A^{\prime}, N$ are concyclic, and since MAN, MA'N are equal angles, $M, A, A^{\prime}, N$ are concyclic. Therefore $\Pi, M, A, N$ are concyclic. Therefore MIN, $m \Pi n$ are equal angles. Similarly it may be shown that NII, $n \Pi l$ are equal angles, and also LIM, $l \Pi m$. Therefore $\mathrm{LIl}, \mathrm{MI} m, \mathrm{~N} I n$ are equal angles. Hence it might easily be shown that the triangle LMN is similar to the triangle $/ m n$. Therefore, by Art. 8, its circumcircle forms, in its various positions, a coaxaloid system having $\Pi$ as one of the $\Pi$-points.

31. If a number of coaxaloid systems have the same ratio $r$, and have besides a common $\Pi$-point, $\Pi$, while the other $\Pi$-points lie on any locus, say a straight line, they form a web of circles possessing the following obvious properties.

(i) If a transversal be drawn in any direction across the web, the circles whose centres lie on it form a coaxaloid system one of whose $\Pi$-points is $\Pi$, and whose ratio is $r$; for if the transversal pass through $K_{1}, K_{2}, K_{3} \ldots$ centres of circles of the web, and $\rho K$ denote the radius of the circle whose centre is $K$, the ratios $\rho \mathrm{K}_{1} / \mathrm{K}_{1} \Pi, \rho \mathrm{K}_{2} / \mathrm{K}_{2} \Pi, \rho \mathrm{K}_{3} / \mathrm{K}_{3} \Pi \ldots$ are each equal to $r$.

(ii) When the transversal is drawn through $\Pi$, the circles whose centres lie on it have $\Pi$ as a common centre of similitude; for if $\mathbf{M}_{1}, \mathbf{M}_{2}, \mathbf{M}_{3} \ldots$ be centres lying on the transversal,

$$
\rho M_{3} / M_{1} \Pi=\rho M_{2} / M_{2} \Pi=\ldots=r \text {. }
$$


(iii) The centres of equal circles of the web lie on a circle whose contre is $\Pi$; for if $\mathrm{C}_{1}, \mathrm{C}_{2}, \mathrm{C}_{3} \ldots$ be centres of equal circles,

$$
\rho \mathrm{C}_{1} / \mathrm{C}_{1} \Pi=\rho \mathrm{C}_{2} / \mathrm{C}_{2} \Pi=\rho \mathrm{C}_{3} / \mathrm{C}_{3} \Pi=\ldots=r,
$$

and therefore since $\quad \rho \mathrm{C}_{1}=\rho \mathrm{C}_{2}=\rho \mathrm{C}_{3}=$ etc.,

$$
\mathrm{C}_{1} \Pi=\mathrm{C}_{2} \Pi=\mathrm{C}_{3} \mathrm{II}=\text { etc. }
$$

A web such as has been described may be called a coaxaloid web of the first kind. It may be generated in a great number of different ways, of which the two following may be mentioned.

(1) If a variable triangle of constant species have a fixed point II invariably connected with it, a circle invariably connected with it, forms, in its different positions, a coaxaloid web of the first kind; for, obviously, the radius of the circle bears a constant ratio to the distance of its centre from $\Pi$.

(2) If in the system of triangles referred to in the last article, different triangles be taken in succession as the fixed triangle, the coaxaloid systems so obtained form a web of the first kind.

Demonstration. The centre of similitude, $\Pi$, of the triangles is invariably connected with them. Therefore its pedal triangle with respect to each of them is of constant species. Therefore the triangle determined by the intersections of homologous sides of every pair of them is also of constant species, and has $\Pi$ invariably connected with it. Therefore by (1) above, its circumcircle forms a web of the first kind.

32. If a vuriable line terminated at $\Pi$ and $\Pi^{\prime}$ move parallel to itself so that $\Pi$ and $\Pi^{\prime}$ always lie on two fixed straight lines intersecting in $\mathrm{S}$, a coaxaloid system of circles having $\Pi$ and $\Pi^{\prime}$ as $\Pi$-points and a constant ratio $r$, generates, as $\Pi \Pi^{\prime}$ varies, a web of circles which may be called a coaxaloid web of the second kind, and which possesses the following properties.

(i) Those circles of the web whose centres lie on any transversal through $\mathrm{S}$, have $\mathrm{S}$ as a common centre of similitude.

(ii) Generally the circles whose centres lie on any transversal are coaxaloid.

(iii) The coaxaloid systems determined by parallel transversals have the same ratio, and have their $\Pi$-points lying on two straight lines through $\mathbf{S}$. 
(iv) Systems of equal circles belonging to the web have their centres lying on a series of similar and similarly placed ellipses with $\mathrm{S}$ as centre.

Demonstration. Let $\Pi_{1} \Pi_{1}^{\prime}$ and $\Pi_{2} \Pi_{2}^{\prime}$ (Fig. 15) be two positions of $\Pi \Pi \Pi^{\prime}$, and let $\mathrm{O}_{1}, \mathrm{O}_{2}$ be their respective mid-points. Let the perpendiculars at $O_{1}, O_{2}$ to $\Pi_{1} \Pi_{1}^{\prime}$ and $\Pi_{2} \Pi_{2}^{\prime}$ (the lines of centres of the coaxaloid systems whose $\Pi$-points are $\Pi_{1}, \mathrm{II}_{1}^{\prime}$ and $\Pi_{2}, \Pi_{2}^{\prime}$ respectively), meet a transversal through $S$ in $\mathrm{C}_{1}, \mathrm{C}_{3}$, and a parallel transversal in $K_{1}, K_{2}$ respectively. Through $S$ draw a parallel to $\mathrm{O}_{1} \mathrm{~K}_{1}$, meeting $\mathrm{K}_{1} \mathrm{~K}_{2}$ in $\mathrm{K}$. It will be noticed that $\mathrm{SK}$ is the line of centres of that one of the original coaxaloid systems whose II-points coincide in S. Let $\rho \mathrm{C}$ denote the radius of the circle (belonging to the web) whose centre is $\mathrm{C}$.

(i) $\mathrm{C}_{1} \mathrm{~S} / \mathrm{C}_{2} \mathrm{~S}=\mathrm{O}_{1} \mathrm{~S} / \mathrm{O}_{2} \mathrm{~S}=\Pi_{1} \mathrm{~S} / \Pi_{2} \mathrm{~S}$.

Therefore $C_{1} \Pi_{1}$ and $C_{.} \Pi_{2}$ are parallel.

Therefore $\mathrm{C}_{1} \Pi_{1} / \mathrm{C}_{1} \mathrm{~S}=\mathrm{C}_{2} \Pi_{2} / \mathrm{C}_{2} \mathrm{~S}$.

But $\rho \mathrm{C}_{1} / \mathrm{C}_{1} \Pi_{1}=\rho \mathrm{C}_{2} / \mathrm{C}_{2} \Pi_{2}=r$.

Therefore $\rho \mathrm{C}_{1} / \mathrm{C}_{1} \mathrm{~S}=\rho \mathrm{C}_{2} / \mathrm{C}_{2} \mathrm{~S}$.

Therefore $S$ is the centre of similitude of the circles $C_{1}$ and $C_{2}$. In the same way it may be proved to be the centre of similitude of every other pair of circles whose centres lie on the same transversal.

(ii) Let $S K, \Pi_{1} K_{1}$ meet at $Q$, and let the circumcircles of $\mathrm{QSI}_{1}, \mathrm{QKK}_{1}$ meet again in $\mathrm{P}$. Then since $\mathrm{P}, \mathrm{K}, \mathrm{K}_{1}, \mathrm{Q}$ are concyclic, $\mathrm{PKS}$ and $\mathrm{PK}_{1} \Pi_{1}$ are equal angles and since $\mathrm{P}, \mathrm{S}, \Pi_{1}, \mathrm{Q}$ are concyclic, $P S K$ and $P \Pi_{1} K_{1}$ are equal angles. Therefore PKS and $\mathrm{PK}_{1} \Pi_{1}$ are similar triangles.

Hence $\mathrm{PKK}_{1}$ and $\mathrm{PSII}_{1}$ are similar triangles, and, therefore, since $\mathrm{KK}_{1} / \mathrm{KK}_{2}=\mathrm{S} \Pi_{1} / \mathrm{SH}_{2}, \quad \mathrm{PKK}_{2}$ and $\mathrm{PSH}_{2}$ are similar triangles. Hence $\mathrm{PKS}$ and $\mathrm{PK}_{2} \Pi_{2}$ are similar triangles. - . - - (2)

Therefore, by (1) and (2) KS/KP $=K_{1} \Pi_{1} / K_{1} P=K_{2} \Pi_{2} / K_{2} P$.

But $\rho \mathrm{K} / \mathrm{KS}=\rho \mathrm{K}_{1} / \mathrm{K}_{1} \Pi_{1}=\rho \mathrm{K}_{2} / \mathrm{K}_{2} \Pi_{2}=r$.

Therefore $\rho \mathrm{K} / \mathrm{KP}=\rho \mathrm{K}_{1} / \mathrm{K}_{1} \mathrm{P}=\rho \mathrm{K}_{2} / \mathrm{K}_{2} \mathrm{P}$.

Therefore the circles (of the web) whose centres lie on the tranversal $\mathrm{KK}_{1}$ are coaxaluid, with $P$ as a II-point.

(iii) $\mathrm{K} \widehat{\mathrm{P}} \mathrm{K}_{1}=\widehat{K} \widehat{\mathbf{Q K}} \mathrm{K}_{1}=\mathrm{C}_{1} \widehat{\mathrm{K}}_{1} \Pi_{1}$,

and $\mathrm{PK} / \mathrm{PK}_{1}=\mathrm{KS} / \mathrm{K}_{1} \mathrm{II}_{1}=\mathrm{K}_{1} \mathrm{C}_{1} / \mathrm{K}_{1} \Pi_{1}$. 
Therefore $\mathrm{PKK}_{1}$ and $\mathrm{K}_{1} \mathrm{C}_{1} \Pi_{1}$ are similar triangles.

Therefore

$$
\mathrm{PK} / \mathrm{K}_{1} \mathrm{C}_{1}=\mathrm{KK}_{1} / \mathrm{C}_{1} \Pi_{1} \text {, }
$$

$$
\text { i.e. } \mathrm{PK} / \mathrm{K} \mathrm{S}=\mathrm{SC}_{1} / \mathrm{C}_{1} \Pi_{1} \text {. }
$$

But

$$
\mathrm{PK} / \mathrm{PS}=\mathrm{KK}_{1} / \mathrm{S \Pi}_{1}=\mathrm{SC}_{1} / \mathrm{S \Pi}_{1} \text {. }
$$

Therefore $\mathrm{PKS}$ and $\mathrm{SC}_{1} \Pi_{1}$ are similar triangles.

Let $r^{\prime}$ denote the ratio of the system of circles $C_{1}, C_{2} \ldots$, and $r^{\prime \prime}$ the ratio of the system $\mathrm{K}, \mathrm{K}_{1}, \mathrm{~K}_{2} \ldots$.

Then $\rho \mathrm{C}_{1} / \mathrm{C}_{1} \Pi_{1}=r$, and $\rho \mathrm{C}_{1} / \mathrm{C}_{1} \mathrm{~S}=r^{\prime}$.

Therefore $r^{\prime}=r . \mathrm{C}_{1} \Pi_{1} / \mathrm{C}_{1} \mathrm{~S}$.

And $\rho \mathrm{K} / \mathrm{KS}=r$, and $\rho \mathrm{K} / \mathrm{KP}=r^{\prime \prime}$.

Therefore $r^{\prime \prime}=r . \mathrm{KS} / \mathrm{KP}$.

But $\mathbf{K S} / \mathrm{KP}=\mathrm{C}_{1} \Pi_{1} / \mathrm{C}_{1} \mathrm{~S}$.

Therefore $r^{\prime \prime}=r^{\prime}$.

Hence systems whose centres lie on parallel transversals have the same ratio.

Again, since $\mathrm{PKS}$ and $\mathrm{SC}_{1} \Pi_{1}$ are similar triangles, the angle $\mathrm{KSP}$ is equal to the angle $\mathrm{C}_{1} \Pi_{1} \mathrm{~S}$ which is constant so long as $\mathrm{SC}_{1}$ retains its direction. Therefore the locus of $P$, as $K_{1}$ moves parallel to $\mathrm{SC}_{1}$ is a straight line through $\mathrm{S}$. Hence it may be readily proved that the locus of the image of $\mathrm{P}$ with respect to $\mathrm{KK}_{1}$, i.e., the other $\Pi$-point, is also a straight line through $\mathrm{S}$. Thus statement (iii) is completely proved.

(iv) Let $K_{1}$ be the centre of a variable circle of the web, having a constant radius $\rho$; and regard the circle as belonging in its different positions to the variable coaxaloid system whose line of centres is parallel to $S K$, and of which $\Pi_{1}, O_{1}$ (here regarded as variable) are respectively a II-point, and the mid-point of the join of the $\Pi$-points. Let $\alpha$ denote the angle $\Pi_{1} \mathrm{SO}_{1}$ and $\beta$ the angle $\mathrm{SH}_{2} \mathrm{O}_{2}$.

Then

$$
\begin{aligned}
& \mathrm{K}_{1} \Pi_{3}=\rho / r, \\
& \mathrm{~K}_{1} \Pi_{1}^{2}=\mathrm{K}_{1} \mathrm{O}_{1}^{2}+\Pi_{1} \mathrm{O}_{1}^{2}, \\
& \Pi_{1} \mathrm{O}_{1}=\frac{\sin a}{\sin \beta} \cdot \mathrm{SO}_{1} \\
& \mathrm{~K}_{1} \mathrm{O}_{1}^{2}+\frac{\sin ^{2} \alpha}{\sin ^{2} \beta} \cdot \mathrm{SO}_{1}^{2}=\frac{\rho^{2}}{r^{2}} .
\end{aligned}
$$

and

Therefore

Therefore, taking $\mathrm{SO}_{1}$ and $\mathrm{SK}$ as the axes of $x$ and $y$ respectively, 
we have as the equation of the locus of $K_{1}$,

$$
y^{2}+\frac{\sin ^{2} a}{\sin ^{2} \beta} \cdot x^{2}=\frac{\rho^{2}}{r^{2}},
$$

which represents an ellipse having $S$ as centre, and a pair of conjugate diameters coincident with $\mathrm{SO}_{1}$ and $\mathrm{SK}$. By making $\rho$ vary, we obtain a series of concentric, similar and similarly placed ellipses. Thus statement (iv) is proved.

It may be noted that in the case when $S \Pi=S \Pi$, the lines of centres (SK, $\mathrm{O}_{1} \mathrm{~K}_{1}, \mathrm{O}_{2} \mathrm{~K}_{2}$, etc.) of the generating systems coincide, so that the web obtained is such that any point on $\mathrm{SO}_{1}$ is the centre of a concentric system.

A coaxaloid web of the second kind may be generated in a great many different ways, of which the following one may be mentioned. In connection with Fig. 6 it was shown that if $\triangle B C$ remain fixed, while PQR varies so as to remain homothetic with itself with respect to $\mathrm{S}$ as homothetic centre, we obtain a coaxaloid system $I 2 \mathrm{M} m \mathrm{~N} n$ connected with $\mathrm{ABC}$. If now $\mathrm{ABC}$ also be made to vary so as to remain homothetic with itself with respect to $S$ as homothetic centre, we obtain a series of coaxaloid systems having the same S-point, and forming a web of the second kind; for as may easily be proved by similar triangles, their ratios are the same, their II-points lie on two straight lines intersecting in $S$, and their line of centres are either parallel or, as is the case when the $\Pi$-points are the Brocard points of $\mathrm{ABC}$, coincident.

\section{Note referring to Art. 17.}

Let $\mathrm{K}$ be the centre (Fig. 12) and TT' the diameter through $I I$ and $I^{\prime}$ of the minimum circle of a cosxaloid systen whose $\Pi$-points (real) are $\Pi$ and $\Pi$. On the line of centres of the system, on opposite sides of $K$, take two points $P$ and $P^{\prime}$ such that $\mathrm{KP}=\mathrm{KP}=\mathrm{K \Pi}$. Suppose that a system of coaxal circles is constructed with TT' $^{\prime}$ as radical axis, and $P, P^{\prime}$ as limiting points ; and suppose that the radii of these are increased or diminished, as the case may be, in the ratio $\mathrm{KT} / \mathrm{K} \Pi$, denoted by $r$, so as to give rise to a coaxaloid system with iuaginary $\Pi$-points. Let $K^{\prime}$ be the centre of any circle of this system, and $K^{\prime} W$ a radius of it parallel to any fixed direction $K V$. Draw $K^{\prime} R$ a radius of the circle of the 
original coaxal system, whose centre is $K^{\prime}$, parallel to 'TT'; and draw RM perpendicular to 'TT', and join MP, M.K'. Then if $x$ and $y$ be the coordinates of $W$, with respect to $K V, K P$ as axes, we have

$$
x^{2}=\mathrm{K}^{\prime} \mathrm{W}^{2}=r^{2} \mathrm{~K}^{\prime} \mathrm{R}^{2}=r^{\prime}\left(\mathrm{K}^{\prime} \mathrm{M}^{2}-\mathrm{RM}^{2}\right) \text {. }
$$

But $R M=P M$, both being tangents from a point on the radical axis to circles of the coaxal system. Therefore

$$
x^{2}=r^{2}\left(\mathrm{~K}^{\prime} \mathrm{M}^{2}-\mathrm{PM}^{2}\right)=r^{2}\left(\mathrm{~K}^{\prime} \mathrm{K}^{2}-\mathrm{PK}^{2}\right)=\left(y^{2}-\mathrm{K}^{2}\right) \mathrm{KT}^{2} / \mathrm{K} \Pi^{2} .
$$

Hence the equation of the locus of $\mathrm{W}$ is

$$
y^{2} / \mathbf{K} \Pi^{2}-x^{2} / \mathrm{KT}^{2}=1 \text {. }
$$

It will be noticed that the hyperbolas in this case are the conjugates of those associated with the coaxaloid system whose $\Pi$-points (real) are $\Pi$ and $\Pi^{\prime}$, and whose ratio is also $\mathrm{KT} / \mathrm{K} \Pi$.

It may be remarked that the foregoing proof and that of Art. 17 implicitly contain the following theorem which I have not seen stated elsewhere: If $K^{\prime} W$ (Fig. 5) be a variable line of fixed direction, such that its extremity $\mathbf{K}^{\prime}$ moves on a fixed line ( $\mathbf{K} \mathbf{K}^{\prime \prime}$ ) and such that $K^{\prime} W / K^{\prime} \Pi$, where $\Pi$ is a fixed point, is constant, the locus of $\mathbf{W}$ is a hyperbola.

Systems of Conics connected with the Triangle.

$$
\text { By J. A. Third, M.A. }
$$

[The references in square brackets are to the articles of my paper on Systems of Circles anslogous to Tucker Circles.]

I.

Systems of Six-point Conics.

1. It has been shown [4] that if two triangles $A B C$ and $P Q R$ (Fig. 6) be in perspective with respect to any point $S$ as centre of perspective, the sides of $P Q R$ cut the non-corresponding sides of $A B C$ in three pairs of points which lie on a circle, provided that the angles made by the sides of $\mathrm{PQR}$ with those of $\mathrm{ABO}$ possess certain 